\title{
The impacts of surface conditions on the vapor-liquid-solid growth of germanium nanowires on Si (100) substrate
}

\author{
C. B. Li, ${ }^{1,4, a)}$ K. Usami, ${ }^{1,4}$ T. Muraki, ${ }^{1}$ H. Mizuta, ${ }^{2,3,4}$ and S. Odal ${ }^{4}$ \\ ${ }^{1}$ Quantum Nanoelectronics Research Center, Tokyo Institute of Technology, Tokyo 152-8552, Japan \\ ${ }^{2}$ School of Electronics and Computer Science, The University of Southampton, Highfield, Southampton, \\ Hampshire SO17 1BJ, United Kingdom \\ ${ }^{3}$ Department of Physical Electronics, Tokyo Institute of Technology, Tokyo 152-8552, Japan \\ ${ }^{4}$ Solution-Oriented Research for Science and Technology, Japan Science and Technology (SORST-JST), \\ Japan
}

(Received 27 June 2008; accepted 15 July 2008; published online 1 August 2008)

\begin{abstract}
The impacts of surface conditions on the growth of Ge nanowires on a $\mathrm{Si}(100)$ substrate are discussed in detail. On $\mathrm{SiO}_{2}$-terminated $\mathrm{Si}$ substrates, high-density Ge nanowires can be easily grown. However, on H-terminated Si substrates, growing Ge nanowires is more complex. The silicon migration and the formation of a native $\mathrm{SiO}_{2}$ overlayer on a catalyst surface retard the growth of Ge nanowires. After removing this overlayer in the HF solution, high-density and well-ordered Ge nanowires are grown. Ge nanowires cross vertically and form two sets of parallel nanowires. It is found that nanowires grew along $\langle 110\rangle$ directions. (C) 2008 American Institute of Physics.
\end{abstract} [DOI: $10.1063 / 1.2968201]$

One-dimensional semiconductor nanostructures have attracted much attention because of their potential applications in the design of nanoelectronic, photonic, and sensing devices. ${ }^{1}$ Due to their high mobility of electrons and holes, Ge nanowires show their potential application in high-speed field-effect transistors. ${ }^{2-4}$ Moreover, Ge nanowires are potentially useful for high-speed quantum computing because of long decoherence time due to the predominance of spin-zero $^{5,6}$ nuclei and the advantage of a large excitonic Bohr radius in $\mathrm{Ge}(24.3 \mathrm{~nm})$, allowing for quantum confinement to be observed in relatively large structures ${ }^{7,8}$ and at high temperatures. In order to realize these applications, controllable and high-quality nanowire growth is important. Much attention has been focused on the growth of $\mathrm{Ge}$ nanowires. ${ }^{9-12}$ However, only few papers discuss the impacts of surface conditions on the vapor-liquid-solid synthesis of Ge nanowires. In this letter, we will discuss them in detail.

Au catalyst layers with thicknesses of 0.1 and $1 \mathrm{~nm}$ were evaporated by electron beam evaporation at room temperature on two kinds of substrates: $\mathrm{SiO}_{2}$-terminated (a few nanometers of native $\mathrm{SiO}_{2}$ layer or $170 \mathrm{~nm}$ thermal oxidized $\mathrm{SiO}_{2}$ ) and $\mathrm{H}$-terminated (the wafers were dipped in $1.5 \% \mathrm{HF}$ solution for $1 \mathrm{~min}$ and were immediately loaded in the chamber for electron beam evaporation) silicon (100) substrates. In order to satisfy the rules of minimum surface energy, the evaporated $\mathrm{Au}$ shows different topographies at different substrate surface conditions, as shown in Figs. 1(a)-1(d). On the $\mathrm{SiO}_{2}$-terminated substrate, because the condensing $\mathrm{Au}$ adatoms are more strongly bound to each other than to the substrate, ${ }^{13}$ these atoms encounter other atoms, nucleate, and agglomerate to form stable islands with diameters of 2-10 nm [Figs. 1(a) and 1(b)]. The thicker Au layer will induce the formation of bigger Au dots. On the other hand, on the H-terminated $\mathrm{Si}$ substrate, the $\mathrm{Au}$ catalyst prefers to deposit in the Stranski-Krastanov mode to form $\mathrm{Au}$ small

\footnotetext{
a) Tel.: +81-(0)3-5734-2542. FAX: +81-(0)3-5734-2542. Electronic mail: cbli@neo.pe.titech.ac.jp.
}

islands with a wetting layer, as shown in Figs. 1(c) and 1(d). All Ge nanowires were grown at $300{ }^{\circ} \mathrm{C}$ for $20 \mathrm{~min}$ by a low-pressure chemical vapor deposition method with $10 \%$ $\mathrm{GeH}_{4}$ precursors (in an atmosphere of hydrogen) in a total pressure of 5 Torr. On a $\mathrm{SiO}_{2}$-terminated $\mathrm{Si}$ substrate, without any pretreatment to the catalysts, high-density Ge nanowires with diameters of 5-20 nm were grown on Au catalysts with thicknesses of $1 \mathrm{~nm}$ (Fig. 2) and $0.1 \mathrm{~nm}$. Both high-resolution transmission electron microscopy and $\mathrm{x}$-ray diffraction results reveal high-quality single-crystalline $\mathrm{Ge}$ nanowires with a cubic diamond structure as we discussed before. ${ }^{14}$ It was found that the thickness of the $\mathrm{SiO}_{2}$ layer, either a few nanometers of native layer or a thicker thermal oxidized $\mathrm{SiO}_{2}$, has no influence on the growth of Ge nanowires.

However, very few Ge nanowires were grown on the H-terminated Si substrate under the same growth conditions (Fig. 3). Even after high temperature preannealing $\left(650^{\circ} \mathrm{C}\right.$ in vacuumed condition) to dewet the Au wetting layer, it had no great influence on the growth of Ge nanowires.

When observing the topography of Au catalysts evaporated on the H-terminated substrate by a scanning electron microscope (SEM), we noticed that the contrast between the

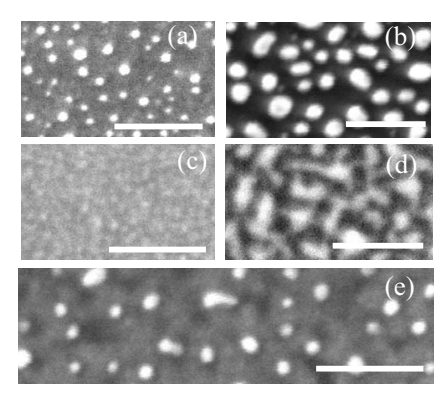

FIG. 1. SEM images of Au catalysts evaporated on $\mathrm{SiO}_{2}$-terminated silicon substrates with a thickness of (a) $0.1 \mathrm{~nm}$ and (b) $1 \mathrm{~nm}$. Au catalysts evaporated on $\mathrm{H}$-terminated silicon substrates with a thickness of (c) $0.1 \mathrm{~nm}$ and (d) $1 \mathrm{~nm}$. (e) SEM image of 1-nm-thick Au evaporated on H-terminated silicon after $\mathrm{HF}$ treatment for $2 \mathrm{~min}$. The scale bars in the figures are $30 \mathrm{~nm}$. 


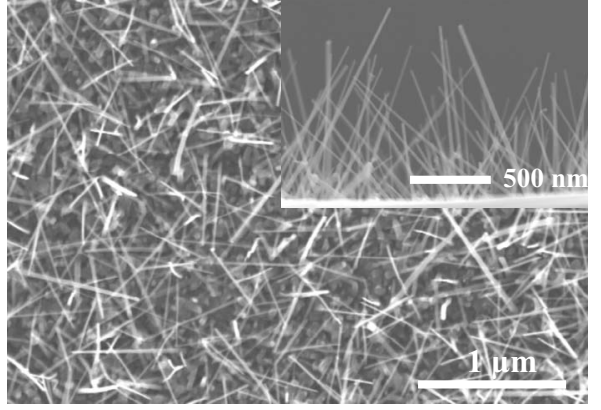

FIG. 2. Top-view SEM image of high-density Ge nanowires grown on 1-nm-thick $\mathrm{Au}$ catalysts evaporated on $\mathrm{SiO}_{2}$-terminated silicon substrates. The inset shows their cross-section image.

Au metal and the Si substrate at the edge area is smaller and the brightness of the Au metal in the SEM image is weaker compared with that evaporated on the $\mathrm{SiO}_{2}$ substrate. It seems that $\mathrm{Au}$ catalysts are covered by some insulator layers. So maybe the formation of a very thin silicon oxide overlayer due to the gold catalyzed migration of silicon through the gold film ${ }^{15-18}$ retards the growth of Ge nanowires.

In order to verify this hypothesis, Au catalysts evaporated on $\mathrm{H}$-terminated substrates were dipped into a $1.5 \% \mathrm{HF}$ solution for $2 \mathrm{~min}$ and then were immediately loaded into a growth chamber to grow $\mathrm{Ge}$ nanowires. On 1-nm-thick $\mathrm{Au}$ catalyst substrates, high-density and well-ordered Ge nanowires were grown, as shown in Fig. 4(a). Obviously, it has a great improvement for Ge nanowire growth by removing this overlayer, and it confirms that the $\mathrm{SiO}_{2}$ overlayer formed on the catalyst surface prevents the growth of Ge nanowires. Figure 1(e) shows the SEM image of 1-nm-thick Au catalysts after $\mathrm{HF}$ treatment. Clear $\mathrm{Au}$ dots can be found after removing the $\mathrm{SiO}_{2}$ overlayer. In the case of 0.1-nm-thick $\mathrm{Au}$ catalysts, even after HF treatment, no nanowire was grown. We did not find any Au dots after HF treatment in the SEM image since they are still in the wetting layer stage. So there are no nucleation centers for Ge atoms to grow in the axial direction. The $\mathrm{GeH}_{4}$ will get more chances to be decomposed and deposited to form a film since the AuSi eutectic alloy exists on the whole surface.

Compared with the random-directed Ge nanowires grown on the $\mathrm{SiO}_{2}$-terminated substrate, as shown in Fig. 2, Ge nanowires grown on the H-terminated Si (100) substrate show a more ordered structure. Almost all Ge nanowires cross vertically and form two sets of parallel nanowires. It should be pointed out that each set of Ge nanowires has around $45^{\circ}$ angle with a [110] cleavage direction as labeled in Fig. 4(a). Until now, only two growth directions, [110] and

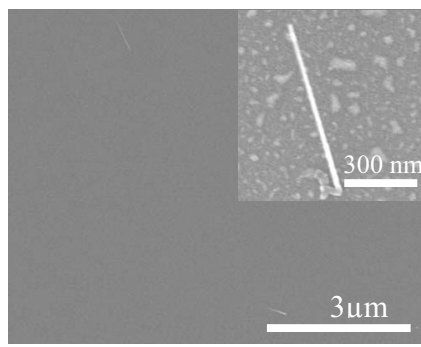

FIG. 3. Top-view SEM image of Ge nanowires grown on H-terminated silicon substrates. Inset shows the enlarged image of one wire, and it exhibits the same diameter as that grown on $\mathrm{SiO}_{2}$-terminated substrates.
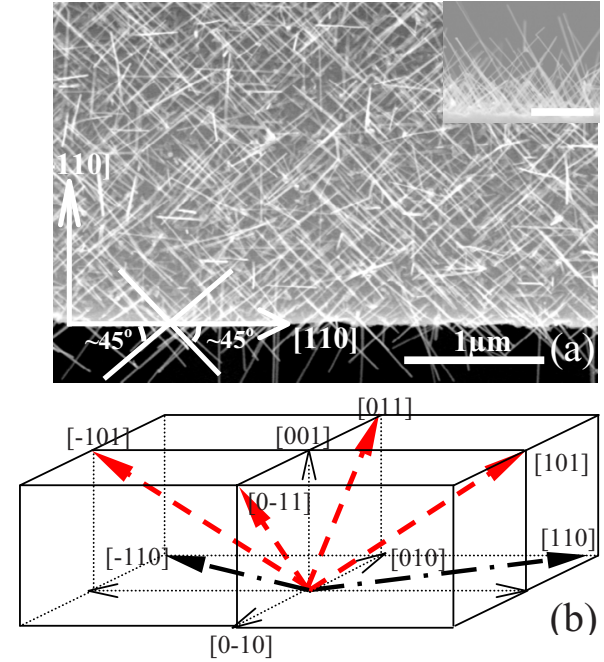

FIG. 4. (Color online) (a) Top-view SEM image of high-density and wellordered $\mathrm{Ge}$ nanowires grown on 1-nm-thick $\mathrm{Au}$ catalysts evaporated on H-terminated silicon (100) after being dipped into the HF solution to remove the $\mathrm{SiO}_{2}$ overlayer. The bottom edge is the [110] cleavage direction. The inset shows its side-view SEM image, and the scale bar is $500 \mathrm{~nm}$. (b) Three-dimensional schematic of the Ge nanowire growth direction on a $\mathrm{Si}$ (100) wafer. Four dashed arrows directed to [101], [011], [-101], and $[0-11]$ are the Ge nanowire growth directions.

[111], were found for $\mathrm{Ge}$ nanowires grown on silicon substrates. ${ }^{2,3,5,10-12}$ Based on this fact and our experiment results, we believe that Ge nanowires are grown orderly along [101], [011], [-101], and [0-11] directions as the four dashed arrows shown in Fig. 4(b), in which these four growth directions are crossed vertically and have a $45^{\circ}$ degree with [110] and [-110] cleavage directions. It agrees well with the experiment results as shown in Fig. 4(a). The two dashed-dotted arrows in Fig. 4(b) are [110] and [-110] cleavage directions for (100) wafer as labeled in Fig. 4(a).

During the growth of $\mathrm{Ge}$ nanowires on $\mathrm{Au}$ catalysts evaporated on the $\mathrm{H}$-terminated $\mathrm{Si}$ substrate after $\mathrm{HF}$ treatment, $\mathrm{GeH}_{4}$ will decompose in the AuSi eutectic alloy located at the interface between the single-crystalline Si surface and Au layer, and then the supersaturation Ge atom will separate out to grow in the axial direction. This ordered structure at the interface can induce the Ge nanowires to grow along only $\langle 110\rangle$ directions. This capability to control the nanowire growth direction would be very important in engineering the transport characteristics for electrons and holes separately to get the best switching performance of a complementary logic circuit configuration. ${ }^{19}$ Also it is quite attractive for future large-scale nanowire integration.

In the case of $\mathrm{Au}$ catalysts evaporated on the $\mathrm{SiO}_{2}$-terminated substrate, maybe the strong bond between $\mathrm{Si}$ and $\mathrm{O}$ atom retards the migration of silicon to the surface and the formation of the $\mathrm{SiO}_{2}$ overlayer. Therefore Ge nanowires can be grown without HF treatment.

In conclusion, high-density Ge nanowires can be easily grown on $\mathrm{SiO}_{2}$-terminated $\mathrm{Si}$ (100) substrates. However, the $\mathrm{SiO}_{2}$ overlayer formed on the surface of Au catalysts evaporated on the H-terminated $\mathrm{Si}$ substrate prevents the growth of Ge nanowires. After removing this $\mathrm{SiO}_{2}$ overlayer by $\mathrm{HF}$ solution, high-density and well-ordered Ge nanowires can be obtained. Moreover, nanowires can be grown orderly along [101], [011], [-101], and [0-11] directions. 
This work is partially supported by SORST-JST (Japan Science and Technology) and Japan Society for the Promotion of Science (JSPS).

${ }^{1}$ H. J. Fan, P. Werner, and M. Zacharias, Small 2, 700 (2006).

${ }^{2}$ J. Xiang, W. Lu, Y. J. Hu, Y. Wu, H. Yan, and C. M. Lieber, Nature (London) 441, 489 (2006).

${ }^{3}$ G. C. Liang, J. Xiang, N. Kharche, G. Klimeck, M. Charles, C. M. Lieber, and M. Lundstrom, Nano Lett. 7, 642 (2007).

${ }^{4}$ S. M. Sze, Physics of Semiconductor Devices (Wiley, New York, 1981).

${ }^{5}$ Y. J. Hu, H. O. H. Churchill, D. J. Reilly, J. Xiang, C. M. Lieber, and C. M. Marcus, Nat. Nanotechnol. 2, 622 (2007).

${ }^{6}$ A. M. Tyryshkin, S. A. Lyon, T. Schenkel, J. Bokor, J. Chu, W. Jantsch, F. Schaffler, J. L. Truitt, S. N. Coppersmith, and M. A. Eriksson, Physica E (Amsterdam) 35, 257 (2006).

${ }^{7}$ X. H. Sun, G. Calebotta, B. Yu, G. Selvaduray, and M. Meyyappan, J. Vac. Sci. Technol. B 25, 415 (2007).

${ }^{8}$ Y. Maeda, N. Tsukamoto, Y. Yazawa, Y. Kanemitsu, and Y. Masumoto,
Appl. Phys. Lett. 59, 3168 (1991).

${ }^{9}$ L. J. Lauhon, M. S. Gudiksen, D. Wang, and C. M. Lieber, Nature (London) 420, 57 (2002).

${ }^{10}$ D. Wang and H. Dai, Angew. Chem., Int. Ed. 41, 4783 (2002).

${ }^{11}$ A. B. Greytak, L. J. Lauhon, M. S. Gudiksen, and C. M. Lieber, Appl. Phys. Lett. 84, 4176 (2004).

${ }^{12}$ S. Kodambaka, J. Tersoff, M. C. Reuter, and F. M. Ross, Science 316, 730 (2007).

${ }^{13}$ J. A. Venables, J. Vac. Sci. Technol. B 4, 870 (1986).

${ }^{14}$ C. B. Li, K. Usami, H. Mizuta, and S. Oda, IEEE SNW 2008, Honolulu.

${ }^{15}$ A. Hiraki, M. A. Nicolet, and W. J. Mayer Appl. Phys. Lett. 18, 178 (1971).

${ }^{16}$ G. Le Lay, Surf. Sci. 132, 169 (1983).

${ }^{17}$ A. Hiraki, Surf. Sci. Rep. 3, 357 (1984).

${ }^{18}$ H. Jagannathana, Y. Nishi, M. Reuter, M. Copel, E. Tutuc, S. Guhab, and R. P. Pezzia, Appl. Phys. Lett. 88, 103113 (2006).

${ }^{19}$ B. Yu, X. H. Sun, G. A. Calebotta, G. R. Dholakia, and M. Meyyappan, J. Cluster Sci. 17, 579 (2006). 